\title{
NKT cells in HIV-1 infection
}

\author{
Demin $\mathrm{Li}^{1}$, Xiao-Ning $\mathrm{Xu}^{1}$ \\ ${ }^{I}$ MRC Human Immunology Unit, Weatherall Institute of Molecular Medicine, John Radcliffe Hospital, Oxford University, Headington, \\ Oxford OX3 9DS, United Kingdom
}

\begin{abstract}
Natural killer T (NKT) cells are a unique $T$ cell population that have important immunoregulatory functions and have been shown to be involved in host immunity against a range of microorganisms. It also emerges that they might play a role in HIV-1 infection, and therefore be selectively depleted during the early stages of infection. Recent studies are reviewed regarding the dynamics of NKT depletion during HIV-1 infection and their recovery under highly active antiretroviral treatment (HAART). Possible mechanisms for these changes are proposed based on the recent developments in HIV pathogenesis. Further discussions are focused on HIV's disruption of NKT activation by downregulating CD1d expression on antigen presentation cells (APC). HIV-1 protein Nef is found to play the major role by interrupting the intracellular trafficking of nascent and recycling CD1d molecules.
\end{abstract}

Keywords: NKT cells, HIV-1, CD1d downregulation

Cell Research (2008) 18:817-822. doi: 10.1038/cr.2008.85; published online 22 July 2008

\section{Introduction}

Natural killer T (NKT) cells are innate-like T lymphocytes recognising glycolipid antigens presented by the MHC class I-related glycoprotein CD1d, which is a member of the lipid-specific antigen presenting molecules CD1 family $[1,2]$. The CD1 proteins, which also include CD1a, CD1b, $\mathrm{CD} 1 \mathrm{c}$, and CD1e, share sequence homology and overall domain structure with MHC class I molecules, consisting of a heavy chain with three extracellular domains and a non-covalently associated light chain $\beta 2$-microglobulin $(\beta 2 \mathrm{~m})[3,4]$. The antigen binding structures of CD1 members are mainly composed of hydrophobic amino acids, enabling them to bind and present lipid antigens instead of peptides to cognate $T$ cells. The lipid antigens presented by CD1d can be endogenously derived, such as isoglobotrihexosylceramide (iGb3) [5] and ganglioside GD3 [6], or microorganism-derived, like $\alpha$-glucuronosylceramide from Sphingomonas [7], $\alpha$-galactosyldiacylglycerol from Borrelia burgdorferi [8] and phosphatidylinositolmannoside PIM4 from mycobacteria [9]. The strongest CD1d ligand, however, is the synthetic glycosphingolipid $\alpha$-galactosylceramide ( $\alpha$ GalCer) that is a derivative from the extracts

Correspondence: Xiao-Ning $\mathrm{Xu}^{\mathrm{a}}$, Demin $\mathrm{Li}^{\mathrm{b}}$

Tel: +44-1865-222403; Fax: +44-1865-222502

aE-mail: xiaoning.xu@imm.ox.ac.uk

bE-mail: demin.li@imm.ox.ac.uk of a marine sponge Agelas mauritianus [10]. It was the discovery of $\alpha \mathrm{GalCer}$ that first led to the identification of NKT cells [11], and it is still being used broadly in various functional assays and to generate CD1d tetramers for NKT cell detection.

NKT cells are a rare population, comprising only $0.01 \sim$ $1 \%$ of human peripheral blood mononuclear cells (PBMC), and they are unique in that they possess semi-invariant $\mathrm{T}$ cell receptors (TCR) which in humans are composed of a V $\alpha 24-J \alpha 18$ TCR $\alpha$ chain preferentially coupled with a V $\beta 11$ TCR $\beta$ chain. NKT cells are important immunoregulatory cells producing both Th1 and Th2 cytokines, which can affect innate immunity through the recruitment and activation of effector cells, such as NK cells, macrophages, dendritic cells (DCs), as well as conventional CD4+ and CD8+ T cells. NKT cells can also be directly cytolytic through mechanisms believed to involve the perforin/granzymes pathway as well as the Fas/FasL pathway $[12,13]$. Functionally NKT cells can be divided into two subpopulations according to their CD4 expression. CD4+ NKT cells, which compose about $50 \%$ of human NKT cells, secrete both Th1 and Th2 cytokines, and might be oriented toward providing B cell help or immunoregulatory functions; CD4- cells mainly produce Th1 cytokines and have stronger cytolytic activity, therefore they might be more oriented towards cell-mediated effector functions $[14,15]$.

The role of NKT cells in viral immunity has yet to be fully elucidated. However, there is strong evidence indicat- 
ing that NKT cells are involved in the immune response against a range of viruses. Conversely, to counteract the host immunity, viruses have evolved to target NKT cells specifically by using various strategies $[1,16]$. Human immunodeficiency virus (HIV)-1 is one such virus and the role of NKT cells in HIV infection was reviewed by Unutmaz a few years ago [17]. Since then there has been significant progress in the field and this review will focus on the new developments and discuss the mechanisms and implications of NKT cells in HIV-1 infection.

\section{NKT depletion during HIV-1 infection}

The most prominent change with NKT cells observed during HIV-1 infection is their rapid selective depletion from the circulation. Three independent studies convincingly showed that HIV-1 infection caused a significant reduction in NKT levels at a rate faster than that of conventional CD4+ T cells [18-20]. Of the different subpopulations, the CD4+ subset showed faster depletion inversely correlated with viral load, and the CD4- subset showed slower depletion $[18,19]$, implying that different mechanisms are involved.

CD4+ NKT cells are favoured targets for HIV-1 infection. The major receptor for HIV-1 is the surface expressed CD4 and the virus mainly infects CD4-expressing T lymphocytes, monocytes/macrophages and dendritic cells (DC) that also express at least one of the co-receptors CCR5 or CXCR4 [21, 22]. The CD4+ subset of NKT cells are found to express high levels of CCR5 and low levels of CXCR4, which makes them susceptible to infection by viruses preferentially using CCR5 as the co-receptor, or so-called R5-tropic strains [18, 23]. Motsinger et al. proved that NKT cells are much more predisposed to R5-tropic infection than other cell types by showing that GFP-tagged virus could infect up to $50 \%$ of $\alpha$ GalCer-activated CD4+ NKT cells in contrast to $5 \%$ of conventional CD4+ T cells, which conversely are twice as susceptible as CD4+ NKT cells to infection with an X4-tropic virus [18]. R5-tropic virus-infected NKT cells are also more efficient for virus replication than conventional T cells. $\alpha$ GalCer-stimulated NKT cells can produce 10 20 fold more viral particles and 10 fold more infectious virus than SEB-activated conventional T cells [18]. Since R5-tropic viruses are the predominant form during viral transmission and early in the course of the disease, this high susceptibility to infection and high efficiency for viral replication would mean that NKT cells are preferentially targeted during acute and early HIV-1 infection, which may play a role in the establishment of infection.

Different subsets of NKT cells appear to be depleted through different mechanisms during HIV-1 infection.
The research on HIV pathogenesis has made revolutionary discoveries over the past few years and it has become clear that $\mathrm{CD} 4+\mathrm{T}$ cells undergo a biphasic depletion process during the infections of HIV-1 and its close relative simian immunodeficiency virus (SIV) [24]. In the acute phase of infection, the virus destroys most CD4+ T cells of gut-associated lymphoid tissue (GALT), which accounts for most total body CD4 $+\mathrm{T}$ cells, through cytopathic viral lysis or CTL killing [25-29]. Immune depletion consequently causes the breakdown of mucosal barrier function, allowing translocation of bacteria and their products, resulting in non-specific systemic immune activation and lymphocyte activation-induced $\mathrm{T}$ cell death in the chronic phase [30, 31]. The fast depletion of CD4+ NKT cells in the acute phase is likely due to the direct consequence of infection, although their distribution in the GALT under normal condition and HIV-1 infection needs to be addressed to verify the theory. Their depletion in the chronic phase is probably due to activation-induced cell death as well as the continual lytic infection because of their high efficiency for HIV-1 infection. Persistent immune activation in the chronic phase also leads to increased $\mathrm{T}$ cell turnover and thus immunologic senescence as well as preferential loss of naïve cells with accumulation of cells with effector, terminally differentiated phenotypes [32]. CD4-NKT cells are resistant to HIV-1 infection, thus infection-induced cell death is unlikely to have any impact on this population [23], it is probable that inappropriate cell activation is the main reason CD4- NKT cells are eliminated. Nevertheless, this does not exclude other possibilities, such as the preferential redistribution and sequestration in lymphoid tissue driven by inflammatory lymphocyte chemokines produced during HIV infection. CD4- NKT cells have been shown to have high expression of chemokine receptors that are crucial for NKT homing and trafficking, such as CD11a and CCR2 [33, 34].

Because NKT cells have important immunoregulatory functions involved in tumour immunity, microorganism infections and autoimmune diseases [1], the selective depletion of NKT cells during HIV-1 infection on one hand may facilitate the establishment and persistence of the infection, and on the other could contribute to the weakening of the host immunity and consequently contribute to the cause of opportunistic infections that are frequently seen in later stage of the disease.

\section{Effects of antiretroviral therapy on circulating NKT cells}

Highly active antiretroviral therapy (HAART), the use of a combination of anti-retroviral drugs from different classes, has significantly reduced HIV-associated morbid- 
ity and mortality [35]. By suppressing viral replication, it considerably reduces viral load and increases CD4+ T cell count, and facilitates immune reconstitution. Studies on the dynamics of CD4+ T cell recovery have established that HAART results in a rapid first phase increase in memory and activated $\mathrm{T}$ cells in the blood that is mainly due to the release of the previously lymphoid tissue-sequestered lymphocytes, followed by a second prolonged phase characterised by the continuous slow repopulation with newly produced naïve T cells [36-39].

The effects of HAART on circulating NKT cells, however, has not drawn too much attention until recently, when several studies were published in the past two years discussing the dynamics of NKT cell recovery under HAART treatment [33, 40-42]. Certain discrepancies exist among them, apparently owing to the differences in disease stages and treatment regimes of the cohorts, and also due to the fact that NKT cells are such a small and variable population to study. Nevertheless, a few trends can be observed by carefully dissecting the results.

Firstly, in a manner similar to conventional CD4+ T cells, after the initiation of the treatment NKT cells appear to have an early fast phase recovery caused mainly by redistribution of previously tissue-sequestered cells [33]. Within 3 months of HAART treatment, NKT levels were shown to increase in line with conventional CD4+ T cells. However, when NKT subpopulations were investigated it was found that the redistribution is largely caused by the CD4- instead of the CD4+ subset, which is in contrast to what happens to conventional $\mathrm{T}$ lymphocytes in that both CD4+ and CD8+ cells recover simultaneously during this stage [36]. The reasons behind this are unclear, however, recent studies discovered that HIV-1 infection persists in the GALT during effective HAART and consequently leads to incomplete recovery of CD4+ T cells in the GALT as well as in peripheral blood [28, 43]. If the major pool of CD4+ NKT cells also prove to reside in the GALT, and if GALT proves to be the site for the major loss of CD4+ NKT cells during acute infection, then continual chronic infection in the GALT may as well prevent the reconstitution of infection-prone CD4+ NKT cells during HAART, rendering them slower to recover than infection-resistant CD4- NKT cells. The recent discovery that there is a positive correlation between CD4+ NKT cell and conventional CD4+ T cell recovery under HAART suggests this could well be the case although further experimental proofs are needed [42].

After the fast phase recovery, HAART treatment can maintain NKT level and prevent their further depletion [4042]. After a year's treatment, one can possibly see a weak, statistically insignificant [40] or significant [42] increase of NKT levels and improved cell function [41], although overall they remained depressed. This stage of recovery is apparently caused by the gradual expansion of newly produced cells of both subsets. However, the recovery of CD4- subset seems more prominent than that of the CD4+ subset [42], which may be explained by the early heavier loss of CD4+ NKT cells than their CD4- counterparts, and may also be due to the continual infection in the GALT during the chronic phase.

Overall, NKT cell recovery after HAART treatment is slow, with the CD4+ subset showing slower reconstitution than the CD4- subset and the CD4+ T lymphocyte compartment in general. Nevertheless, the revival of both subsets can be boosted by the administration of IL-2 in addition to HAART therapy [40]. It has been found previously that IL-2 administration in HIV-1infected patients decreased activation and proliferation of CD4+ T cells, subsequently reduced the level of activation-induced cell death and the turnover in this population, and led to a sustained increase in both CD4+ cell percentage and number [44, 45]. Literally it means the normally immune enhancing cytokine IL-2 is serving as an immunosuppressant to lower the overall activity of the immune system during chronic infection, consequently to maintain the homeostasis. The enhanced recovery of CD4+ NKT cells may well be the direct consequence of IL-2 activity, whereas its effect on CD4- NKT cells still requires further investigation, although preceding experiment showed that IL-2 administration did lead to CD4- T cell restoration as well, presumably due to the overall improvement of the immune system [46].

\section{HIV impedes NKT activity by down-regulating CD1d expression on APC}

HIV-1 has acquired many strategies to evade the human immune response. On one hand, it maintains a high mutation rate to escape the specific responses that the immune system develops during the infection, on the other it targets many different components of the immune system to weaken the host defence [47]. In the case of NKT immunity, the virus not only causes their selective depletion, but also interferes with their activation by antigen presenting cells (APC) through down-regulating APC's expression of CD1d, the antigen-presenting molecule for NKT cells (Figure 1). We and others independently discovered that the expression of CD1d by APC can be dramatically reduced when the cells are infected with HIV-1 virus, and consequently these cells are less competent in activating NKT cells [48-50].

Although it was initially suggested that the HIV-1 envelope protein gp120 might be responsible for this effect [50], we and others later demonstrated that the Nef protein plays a more important role, because Nef gene transfec- 
tion alone would inhibit CD1d surface expression, and Nef-deficient HIV-1 virus lost the ability to down-regulate CD1d $[48,49]$.

It is well established that Nef uses different mechanisms to down-regulate MHC-I and CD4 expression, in which it induces CD4 internalisation and degradation via clathrin-mediated endocytic pathway, whereas it disrupts the transport of MHC-I to the cell surface by sequestering the newly synthesised protein in the trans-Golgi network $[51,52]$. The way Nef inhibits CD1d surface expression, however, appears to be more complicated, and may involve a combination of both pathways. Firstly, it was found that the tyrosine motif in the cytoplasmic tail of CD1d, which is important for CD1d internalisation, is indispensable for Nef-mediated CD1d down-regulation [48, 49]. Then it was proved that mutation of the CD4-interacting motifs of Nef also disrupted the down-regulation of CD1d. Thirdly membrane-associated Nef was demonstrated to accelerate the endocytosis of CD1d (Figure 1). Taken together, these results suggested that Nef might use a mechanism similar to that of CD4 down-regulation to reduce CD1d surface expression. Meanwhile, the two motifs of Nef protein involved in MHC-I down-regulation are also found to be required for CD1d down-regulation, and CD1d was discovered to co-localise with Nef in the trans-Golgi network, suggesting that $\mathrm{CD} 1 \mathrm{~d}$ could also follow the 'MHC-1 model' [48].

It is intriguing that HIV-Nef needs redundant ways to restrict CD1d expression. Other viruses showing similar properties use simpler methods: herpes simplex virus (HSV) inhibits the recycling of the internalised CD1d back to the cell surface [53], and Kaposi sarcoma-associated herpesvirus (KSHV) accelerates its endocytosis to the endocytic system [54]. It could be speculated that reducing NKT immunity is so important to HIV-1 survival or expansion that extra effort needs to be made to make sure NKT activity is lowered. However, the mechanism by which HIV-1 activates NKT cells, and how NKT cells interfere with HIV-1 pathogenesis have not been elucidated. Likewise, NKT cells' roles in other viral immunity also need further investigation, despite the fact it has long been confirmed that NKT cells are involved in immunity against a variety of viruses, such as coxackievirus, HSV, encephalomyocarditis virus, lymphocytic choriomeningitis virus, and respiratory syncytial virus $[16,55]$. There has not been a single virusderived lipid antigen identified although NKT are found to react to an array of lipid antigens derived from other microorganisms [56]. Work needs to carry on to try to detect

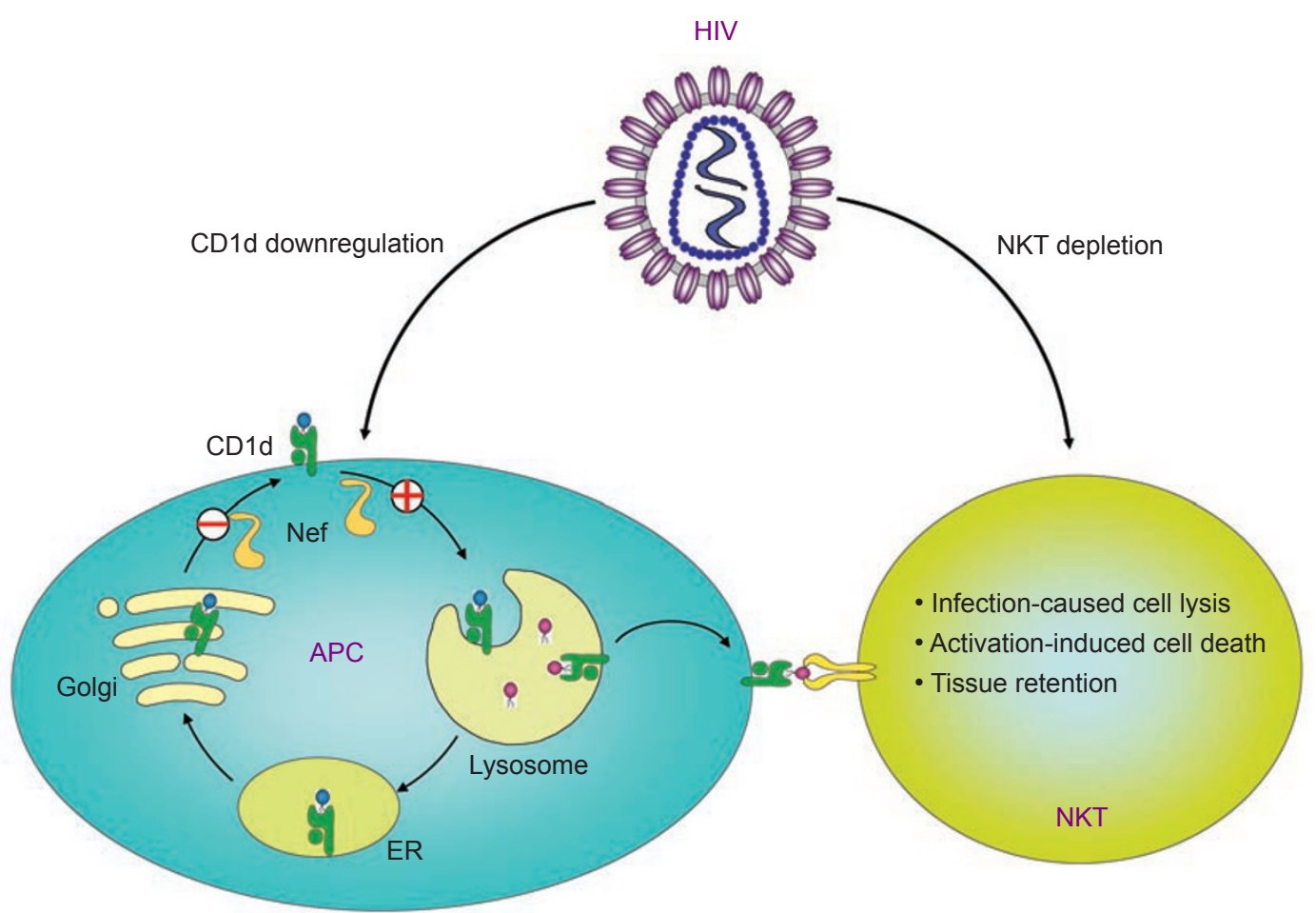

Figure 1 HIV-1 evades NKT function through multiple pathways. On one hand, the virus causes rapid selective NKT depletion by infection-caused cell lysis, activation-induced cell death and inducing retention of NKT in the tissues; on the other, it downregulates CD1d expression on APC by enhancing CD1d internalisation and inhibiting its transportation from the trans-Golgi network to the cell surface, consequently disrupting the activation of NKT cells. 
CD1d-restricted antigens derived from viruses, and at the same time, another possibility also needs to be considered: NKT cells may not recognise any viral antigens, instead, they may be activated indirectly through DC-presented endogenous antigens in combination with cytokines like IL-12 released by activated DC [56]. By down-regulating CD1d levels on DC, the viruses interfere with the interaction between NKT cells and DC, and consequently block one of the major collaborations between innate immunity and adaptive immunity, thereby permitting the viruses to establish persistent infections.

These findings demonstrate that, in addition to classical MHC molecule-mediated antigen presentation, CD1 molecule-mediated lipid antigen presentation is also a frequent target for viral evasion, and improving lipid antigen immune response is a potential area for anti-viral treatment.

\section{Concluding remarks}

NKT cells are an important innate immunity component and have been implicated in immunity against various viral infections, including HIV-1 infection. Consequently NKT cells are selectively depleted by HIV-1 and are prevented from recovery under effective HAART. Meanwhile, the activation of the surviving NKT cells is disrupted by HIV1 Nef protein-mediated CD1d downregulation on APC cells (Figure 1). While Nef is put in the central place in the mechanism for CD1d downregulation, how HIV-1 depletes different subsets of NKT cells are still much down to speculation based on works with conventional $\mathrm{T}$ cells. Further studies are needed to elucidate the distribution of NKT cells in GALT, as well as the relationship between peripheral and GALT-residing NKTs during acute and recovery phases of AIDS conditions.

\section{Acknowledgments}

We thank Drs Sarah Bangs and Tao Dong from the University of Oxford for critical reading of the manuscript, and Medical Research Council for supporting our research.

\section{References}

1 Bendelac A, Savage PB, Teyton L. The biology of NKT cells. Annu Rev Immunol 2007; 25:297-336.

2 Kronenberg M. TOWARD AN UNDERSTANDING OF NKT CELL BIOLOGY: Progress and Paradoxes. Annu Rev Immunol 2005; 23:877-900.

3 Barral DC, Brenner MB. CD1 antigen presentation: how it works. Nat Rev Immunol 2007; 7:929-941.

4 Brigl M, Brenner MB. CD1: antigen presentation and T cell function. Annu Rev Immunol 2004; 22:817-890.

5 Zhou D, Mattner J, Cantu C 3rd, et al. Lysosomal glycosphingo- lipid recognition by NKT cells. Science 2004; 306:1786-1789.

6 Wu DY, Segal NH, Sidobre S, Kronenberg M, Chapman PB. Cross-presentation of disialoganglioside GD3 to natural killer T cells. J Exp Med 2003; 198:173-181.

7 Kinjo Y, Wu D, Kim G, et al. Recognition of bacterial glycosphingolipids by natural killer T cells. Nature 2005; 434:520-525.

8 Kinjo Y, Tupin E, Wu D, et al. Natural killer T cells recognize diacylglycerol antigens from pathogenic bacteria. Nat Immunol 2006; 7:978-986.

9 Fischer K, Scotet E, Niemeyer M, et al. Mycobacterial phosphatidylinositol mannoside is a natural antigen for CD1d-restricted T cells. Proc Natl Acad Sci U S A 2004; 101:10685-10690.

10 Yamaguchi Y, Motoki K, Ueno H, et al. Enhancing effects of (2S,3S,4R)-1-O-(alpha-D-galactopyranosyl)-2-(N-hexacosanoylamino) -1,3,4-octadecanetriol (KRN7000) on antigenpresenting function of antigen-presenting cells and antimetastatic activity of KRN7000-pretreated antigen-presenting cells. Oncol Res 1996; 8:399-407.

11 Kawano T, Cui J, Koezuka Y, et al. CD1d-restricted and TCR-mediated activation of valpha14 NKT cells by glycosylceramides. Science 1997; 278:1626-1629.

12 Metelitsa LS, Naidenko OV, Kant A, et al. Human NKT cells mediate antitumor cytotoxicity directly by recognizing target cell CD1d with bound ligand or indirectly by producing IL-2 to activate NK cells. J Immunol 2001; 167:3114-3122.

13 Kawano T, Nakayama T, Kamada N, et al. Antitumor cytotoxicity mediated by ligand-activated human V alpha24 NKT cells. Cancer Res 1999; 59:5102-5105.

14 Gumperz JE, Miyake S, Yamamura T, Brenner MB. Functionally distinct subsets of CD1d-restricted natural killer T cells revealed by CD1d tetramer staining. J Exp Med 2002; 195:625-636.

15 Lee PT, Benlagha K, Teyton L, Bendelac A. Distinct functional lineages of human V(alpha)24 natural killer T cells. J Exp Med 2002; 195:637-641.

16 Van Dommelen SL, Degli-Esposti MA. NKT cells and viral immunity. Immunol Cell Biol 2004; 82:332-341.

17 Unutmaz D. NKT cells and HIV infection. Microbes Infect 2003; 5:1041-1047.

18 Motsinger A, Haas DW, Stanic AK, et al. CD1d-restricted human natural killer T cells are highly susceptible to human immunodeficiency virus 1 infection. J Exp Med 2002; 195:869-879.

19 Sandberg JK, Fast NM, Palacios EH, et al. Selective loss of innate CD4(+) V alpha 24 natural killer T cells in human immunodeficiency virus infection. $J$ Virol 2002; 76:7528-7534.

20 van der Vliet HJ, von Blomberg BM, Hazenberg MD, et al. Selective decrease in circulating V alpha $24+\mathrm{V}$ beta $11+\mathrm{NKT}$ cells during HIV type 1 infection. J Immunol 2002; 168:1490-1495.

21 Moore JP, Trkola A, Dragic T. Co-receptors for HIV-1 entry. Curr Opin Immunol 1997; 9:551-562.

22 Clapham PR, McKnight A. HIV-1 receptors and cell tropism. $\mathrm{Br}$ Med Bull 2001; 58:43-59.

23 Fleuridor R, Wilson B, Hou R, et al. CD1d-restricted natural killer $\mathrm{T}$ cells are potent targets for human immunodeficiency virus infection. Immunology 2003; 108:3-9.

24 Brenchley JM, Price DA, Douek DC. HIV disease: fallout from a mucosal catastrophe? Nat Immunol 2006; 7:235-239.

25 Mattapallil JJ, Douek DC, Hill B, et al. Massive infection and loss of memory CD4+ T cells in multiple tissues during acute SIV infection. Nature 2005; 434:1093-1097. 
26 Veazey RS, DeMaria M, Chalifoux LV, et al. Gastrointestinal tract as a major site of CD4+ T cell depletion and viral replication in SIV infection. Science 1998; 280:427-431.

27 Brenchley JM, Schacker TW, Ruff LE, et al. CD4+ T cell depletion during all stages of HIV disease occurs predominantly in the gastrointestinal tract. J Exp Med 2004; 200:749-759.

28 Mehandru S, Poles MA, Tenner-Racz K, et al. Primary HIV-1 infection is associated with preferential depletion of CD4+ T lymphocytes from effector sites in the gastrointestinal tract. $J$ Exp Med 2004; 200:761-770.

29 Li Q, Duan L, Estes JD, et al. Peak SIV replication in resting memory CD4+ T cells depletes gut lamina propria CD4+ T cells. Nature 2005; 434:1148-1152.

30 Brenchley JM, Price DA, Schacker TW, et al. Microbial translocation is a cause of systemic immune activation in chronic HIV infection. Nat Med 2006; 12:1365-1371.

31 Appay V, Sauce D. Immune activation and inflammation in HIV-1 infection: causes and consequences. J Pathol 2008; 214:231241.

32 Grossman Z, Meier-Schellersheim M, Sousa AE, Victorino RM,Paul WE. CD4+ T-cell depletion in HIV infection: are we closer to understanding the cause? Nat Med 2002; 8:319-323.

33 van der Vliet HJ, van Vonderen MG, Molling JW, et al. Cutting edge: Rapid recovery of NKT cells upon institution of highly active antiretroviral therapy for HIV-1 infection. $J$ Immunol 2006; 177:5775-5778.

34 Metelitsa LS, Wu HW, Wang H, et al. Natural killer T cells infiltrate neuroblastomas expressing the chemokine CCL2. J Exp Med 2004; 199:1213-1221.

35 Knoll B, Lassmann B, Temesgen Z. Current status of HIV infection: a review for non-HIV-treating physicians. Int J Dermatol 2007; 46:1219-1228.

36 Roederer M. Getting to the HAART of T cell dynamics. Nat Med 1998; 4:145-146.

37 Pakker NG, Notermans DW, de Boer RJ, et al. Biphasic kinetics of peripheral blood $\mathrm{T}$ cells after triple combination therapy in HIV-1 infection: a composite of redistribution and proliferation. Nat Med 1998; 4:208-214.

38 Bucy RP, Hockett RD, Derdeyn CA, et al. Initial increase in blood CD4(+) lymphocytes after HIV antiretroviral therapy reflects redistribution from lymphoid tissues. J Clin Invest 1999; 103:1391-1398.

39 Gorochov G, Neumann AU, Kereveur A, et al. Perturbation of CD4+ and CD8+ T-cell repertoires during progression to AIDS and regulation of the $\mathrm{CD} 4+$ repertoire during antiviral therapy. Nat Med 1998; 4:215-221.

40 Moll M, Snyder-Cappione J, Spotts G, et al. Expansion of CD1drestricted NKT cells in patients with primary HIV-1 infection treated with interleukin-2. Blood 2006; 107:3081-3083.

41 Vasan S, Poles MA, Horowitz A, et al. Function of NKT cells, potential anti-HIV effector cells, are improved by beginning HAART during acute HIV-1 infection. Int Immunol 2007; 19:943951.

42 Yang OO, Wilson SB, Hultin LE, et al. Delayed reconstitution of CD4+ iNKT cells after effective HIV type 1 therapy. AIDS Res Hum Retroviruses 2007; 23:913-922.

43 Chun TW, Nickle DC, Justement JS, et al. Persistence of HIV in Gut-Associated Lymphoid Tissue despite Long-Term Antiretroviral Therapy. J Infect Dis 2008; 197:714-720.

44 Kovacs JA, Lempicki RA, Sidorov IA, et al. Induction of prolonged survival of CD4+ T lymphocytes by intermittent IL-2 therapy in HIV-infected patients. J Clin Invest 2005; 115:21392148.

45 Sereti I, Anthony KB, Martinez-Wilson H, et al. IL-2-induced CD4+ T-cell expansion in HIV-infected patients is associated with long-term decreases in T-cell proliferation. Blood 2004; 104:775-780.

46 Levy Y, Durier C, Krzysiek R, et al. Effects of interleukin-2 therapy combined with highly active antiretroviral therapy on immune restoration in HIV-1 infection: a randomized controlled trial. Aids 2003; 17:343-351.

47 Rowland-Jones SL. Timeline: AIDS pathogenesis: what have two decades of HIV research taught us? Nat Rev Immunol 2003; 3:343-348.

48 Chen N, McCarthy C, Drakesmith H, et al. HIV-1 down-regulates the expression of CD1d via Nef. Eur J Immunol 2006; 36:278286.

49 Cho S, Knox KS, Kohli LM, et al. Impaired cell surface expression of human CD1d by the formation of an HIV-1 Nef/CD1d complex. Virology 2005; 337:242-252.

50 Hage CA, Kohli LL, Cho S, et al. Human immunodeficiency virus gp120 downregulates CD1d cell surface expression. Immunol Lett 2005; 98:131-135.

51 Doms RW, Trono D. The plasma membrane as a combat zone in the HIV battlefield. Genes Dev 2000; 14:2677-2688.

52 Cohen GB, Gandhi RT, Davis DM, et al. The selective downregulation of class I major histocompatibility complex proteins by HIV-1 protects HIV-infected cells from NK cells. Immunity 1999; 10:661-671.

53 Yuan W, Dasgupta A, Cresswell P. Herpes simplex virus evades natural killer $\mathrm{T}$ cell recognition by suppressing CD1d recycling. Nat Immunol 2006; 7:835-842.

54 Sanchez DJ, Gumperz JE, Ganem D. Regulation of CD1d expression and function by a herpesvirus infection. J Clin Invest 2005; 115:1369-1378

55 Skold M, Behar SM. Role of CD1d-restricted NKT cells in microbial immunity. Infect Immun 2003; 71:5447-5455.

56 Tupin E, Kinjo Y, Kronenberg M. The unique role of natural killer $\mathrm{T}$ cells in the response to microorganisms. Nat Rev Microbiol 2007; 5:405-417. 